TRANSACTIONS OF THE

AMERICAN MATHEMATICAL SOCIETY

Volume 287, Number 1, January 1985

\title{
THE DURATION OF TRANSIENTS
}

\author{
BY
}

S. PELIKAN ${ }^{1}$

\begin{abstract}
A transformation $T$ defined on $X \subset \mathbf{R}^{n}$ for which $T(X) \supset X$ is considered. A transient in $X$ is a trajectory $x, T x, \ldots, T^{m} x \subset X$ so that $T^{m+1} x \notin X$. In this case, $m$ is the duration of the transient. A method for estimating the average duration of transients is given, and an example of a transformation with exceedingly long transients is described.
\end{abstract}

Introduction. Imagine a particle moving in a box and making elastic collisions with the sides. Suppose there is a small hole in one side of the box. For many initial conditions the particle will bounce around for a long time and then leave the box. These trajectories are examples of transients. More generally, given a transformation $T$ on $\mathbf{R}^{n}$ which leaves a set $X \subset \mathbf{R}^{n}$ partially invariant (i.e., $T(X) \supset$ $X)$ we call the trajectory through $x$ a transient in $X$ if $x, T x, \ldots, T^{n} x$ all lie in $X$ but $T^{n+1} x \notin X$. In this case $n$ is the duration or lifetime of the transient. In this paper we investigate the average duration of transients for a certain class of transformations $T$.

Transient behavior has been observed in a number of physical systems like the one mentioned above, in the Lorenz model [4], and (recently) in a large class of dynamical systems in which (as a parameter changes) an attractor collides with the boundary of its basin of attraction [2]. Each repeller of a dynamical system has a neighborhood which is partially invariant. Thus we find transient behavior (for the inverse transformation) in the neighborhood of each attractor of a dynamical system.

In $\S 1$ we study conditionally invariant measures. These are tools for the study of transient behavior which were introduced in [3]. In $\S 2$ the average duration of transients is computed in terms of a quantity (the pressure of a function) which, at least in simple cases, can be determined or estimated. Finally, $\S 3$ contains a description of a one-parameter dynamical system for which the average duration of transients is exceedingly long. This example illustrates the effects of factors which determine the duration of transients. It also indicates that great care may be required to distinguish (by means of computer simulation) a system with a chaotic attractor from one with long transients.

1. Measures and transients. Assume that $X$ is a bounded set in $\mathbf{R}^{n}$ and that $T: X \rightarrow \mathbf{R}^{n}$. We will always denote normalized Lebesgue measure on $X$ by

Received by the editors January 5,1984 .

1980 Mathematics Subject Classification. Primary 58F13.

${ }^{1}$ Research sponsored by the Air Force Office of Scientific Research, Air Force Systems Command, USAF, under grant AFOSR-81-0217C. The United States Government is authorized to reproduce and distribute reprints for Government purposes notwithstanding any copyright notation thereon. 
$m$. If $T(X) \supset X$, then $T^{-1}(X)$ is nonempty, as are all the sets $R_{n}=T^{-n}(X)$. The set $\Lambda=\bigcap_{n>0} T^{-n}(X)$ is then nonempty and is, in fact, the maximal $T$-invariant set in $X$. For $x \in X$, let $\tau(x)=\sup \left\{j \geq 0: T^{j}(x) \in X\right\}$. Thus $\tau(x)=n$ if and only if $x \in R_{n} \backslash R_{n+1}$. Then $\tau(x)$ is the duration of the transient beginning at $x$. We are interested in the situations where the expected value of $\tau$, that is $\bar{\tau}=\int_{x} \tau(x) d m$, is finite. This implies that $m$-almost every trajectory leaves $X$ eventually.

Useful tools for the study of transients are conditionally invariant measures, introduced in $[\mathbf{3}]$.

DEFINITION. A probability measure $\mu$ on $X$ is conditionally invariant for $T$ if, for each measurable set $A$,

$$
\mu(A)=\mu\left[T^{-1}(A)\right] / \mu\left[T^{-1}(X)\right] .
$$

This says that the $\mu$-probability that a random point $X$ is in $A$ is the same as the probability that $T(y)$ is in $A$, given that $y$ is a point for which $T(y)$ is in $X$. Note that any $T$-invariant measure is conditionally invariant.

The most important property of conditionally invariant measures is given by

LEMMA 1. Let $T$ be as above and let $\mu$ be a conditionally invariant measure which is not invariant. Then $\mu\left(R_{n}\right)=\alpha^{n}$, where $\alpha=\mu\left(T^{-1} X\right)<1$.

Proof. Because $\mu(A)=\mu\left(T^{-1} A\right) / \mu\left(T^{-1} X\right)$ for any measurable set $A$, we obtain, by taking $A=T^{-n} X$, that

$$
\mu\left(T^{-n} X\right)=\mu\left(T^{-(n+1)} X\right) / \mu\left(T^{-1} X\right) .
$$

Consequently for any $n, \mu\left(T^{-n} X\right) \alpha=\mu\left(T^{-(n+1)} X\right)$, and so $\mu\left(T^{-n} X\right)=\alpha^{n}$.

In the cases studied here, there are more conditionally invariant measures than invariant ones.

The utility of conditionally invariant measures in the study of transients comes from the fact that $\int \tau(x) d \mu$ is easy to evaluate. Since $\tau=n$ on $R_{n} \backslash R_{n+1}$, we obtain from Lemma 1

COROLLARY 1.

$$
\int \tau d \mu=\frac{\alpha}{1-\alpha} \quad \text { and } \quad \int\left(\tau-\int \tau d \mu\right)^{2} d \mu=\frac{\alpha}{(1-\alpha)^{2}}
$$

PROOF. We compute

$$
\int \tau d \mu=\sum_{n=0}^{\infty} n\left[\mu\left(R_{n}\right)-\mu\left(R_{n+1}\right)\right]=\sum_{n=0}^{\infty} n\left(\alpha^{n}-\alpha^{n+1}\right)=(1-\alpha) \sum_{n=0}^{\infty} n \alpha^{n} .
$$

But

$$
\sum_{n=0}^{\infty} n \alpha^{n}=\alpha \sum_{n=0}^{\infty} n \alpha^{n-1}=\alpha\left[\frac{d}{d \alpha} \sum_{n=0}^{\infty} \alpha^{n}\right]=\alpha\left[\frac{d}{d \alpha} \frac{1}{1-\alpha}\right]=\frac{\alpha}{(1-\alpha)^{2}}
$$

hence. $\int \tau d \mu=\alpha /(1-\alpha)$. The proof of the second assertion is a similar computation.

When the measure $\mu$ is equivalent to $m$ (i.e., there is a constant $K \geq 1$ so that $m(A) / K \leq \mu(A) \leq K m(A)$ for each measurable set $A)$, the corollary suffices to estimate $\bar{\tau}$. In $[\mathbf{3}]$ the authors give a condition sufficient to guarantee the existence 
of a conditionally invariant measure $\mu$ equivalent to $m$. This condition is that $T: X \rightarrow \mathbf{R}^{n}$ is expanding and piecewise $C^{2}$. Expanding means that at every point where the derivative $D T(x)$ exists, all its eigenvalues have absolute value greater than 1. Piecewise $C^{2}$ means that $X$ can be written as a finite union of sets $X=X_{1} \cup \cdots \cup X_{k}$ so that the boundary of each $X_{i}$ is smooth, except for a finite number of corners, and so $T$ extends to a $C^{2}$ function on closure $\left(X_{i}\right), i=1, \ldots, k$.

Conditionally invariant measures can be proved to exist under more general conditions than those just described. In particular, consider a diffeomorphism $f$ which has an axiom $A$ attractor $\Lambda$. Let $U$ be a small neighborhood of $\Lambda$ and consider $f^{-1}$ which maps $U$ onto itself: $f^{-1}(U) \supset U$. On each piece of stable manifold of $\Lambda$, i.e., $W^{s}(x) \cap U$, the map $f^{-1}$ acts like an expanding map. A modification of the techniques of $[\boldsymbol{3}]$ can then be used to construct conditionally invariant measures on each leaf $W^{s}(x) \cap U$, and these can be integrated to prove the existence of a conditionally invariant measure for $f^{-1}$.

Unfortunately, the proof of the existence of $\mu$ is not constructive and so the number $\alpha=\mu\left(T^{-1} X\right)$ is not readily attainable. The goal of the next section is to provide another expression for $\alpha$. We begin by recalling the definition of the pressure of a function.

2. Transients and pressure. If $T: \Lambda \rightarrow \Lambda$ is a continuous map of a compact metric space and $\phi: \Lambda \rightarrow \mathbf{R}$ is continuous, the pressure of $\phi$ is defined as follows:

Let $\mathcal{U}=\left\{U_{1}, \ldots, U_{k}\right\}$ be a finite open cover of $\Lambda$. For each $n>0$ denote by $\mathcal{U}^{(n)}$ the collection of all sets of the form

$$
U_{i_{1}} \cap T^{-1} U_{i_{2}} \cap \cdots \cap T^{-(N-1)} U_{i_{n}}=U_{i_{1}, \ldots, i_{n}} .
$$

If the diameter of $\mathcal{U}=\max \operatorname{diameter}\left(U_{j}\right)$ is $\varepsilon$, each set $U_{i_{1}, \ldots, i_{n}}$ in $\mathcal{U}^{(n)}$ represents a collection of points whose $j$ th images under $T$ lie in the same set of $\mathcal{U}$, and hence remain within $\varepsilon$ of each other for $0 \leq j \leq n-1$. The points in $U_{i_{1}, \ldots, i_{n}}$ might then be called $(n, \varepsilon)$ indistinguishable.

When $A \subset U^{(n)}$ is a collection of sets which cover $\Lambda$ we define

$$
Q(\mathcal{A}, \phi, n)=\sum_{A \in \mathcal{A}} \sup _{x \in A} \exp \sum_{j=0}^{n-1} \phi\left(T^{j} x\right)
$$

and

$$
P(\mathcal{U}, \phi, n)=\inf _{\mathcal{A} \text { a subcover }} Q(\mathcal{A}, \phi, n) .
$$

It turns out that the limit

$$
P(\phi, \mathcal{U})=\lim _{n} \frac{1}{n} \log [P(\mathcal{U}, \phi, n)]
$$

exists and is finite. The pressure of $\phi$, denoted $P(\phi)$, is defined by

$$
P(\phi)=\lim _{\text {diameter } u \rightarrow 0} P(\phi, \mathcal{U}) .
$$

Think of $P(\phi, \mathcal{U}, n)$ as the sum of $f=\exp \sum_{j=0}^{n-1} \phi\left(T^{j} x\right)$ over a collection of $(n, \varepsilon)$ indistinguishable orbits in $\Lambda . P(\phi, \mathcal{U})$ is the exponential growth rate of these sums.

If $T$ has a conditionally invariant measure $\mu$ which is equivalent to $m$, then $\alpha=\mu\left(T^{-1} X\right)$ satisfies $\log \alpha=\lim _{n} \frac{1}{n} \log m\left(R_{n}\right)$. This is the idea behind Theorem 1 . 
THEOREM 1. Suppose that $T: X \rightarrow \mathbf{R}^{n}$ is a piecewise $C^{2}$ expanding transformation, where $X=X_{1} \cup \cdots \cup X_{k}$ is a decomposition of $X$ into pieces on which $T$ is $C^{2}$. Suppose $T$ maps each $X_{j}$ onto $X$. Set $\phi(x)=-\log (|\operatorname{det} D T(x)|)$ and let $P(\phi)$ be the pressure of $\phi$ on $T: \Lambda \rightarrow \Lambda$, where $\Lambda$ is the maximal $T$-invariant set in $X$. Then $\log \alpha=P(\phi)$.

Proof. By hypothesis, $R_{n}=T^{-n}(X)$ consists of $k^{n}$ pieces $\left\{C_{j}^{n}\right\}_{j=1}^{k^{n}}$, so that $T^{n}$ maps each $C_{j}^{n}$ onto $X$ and $T^{n} \mid C_{j}^{n}$ is $C^{2}$. We ensure that $T: \Lambda \rightarrow \Lambda$ and $\phi: \Lambda \rightarrow \mathbf{R}$ are continuous by giving $\Lambda$ the topology in which each of the sets $\Lambda_{j}^{n}=\Lambda \cap C_{j}^{n}$ are open.

There are constants $0<\lambda<1$ and $B>0$ so that $\max _{j} \operatorname{diameter}\left(C_{j}^{n}\right) \leq B \lambda^{n}$. To see this, let $B=\operatorname{diameter}(T(X))$ and

$$
\mu=\inf _{1 \leq j \leq k} \inf _{X_{j}}\{\lambda: \lambda \text { is eigenvalue of } D T(x)\}>1 .
$$

If $x, y \in C_{j}^{n}$, then $\operatorname{dist}\left(T^{n} x, T^{n} y\right) \geq \operatorname{dist}(x y) \mu^{n}$. But $T^{n} x$ and $T^{n} y \in T(X)$ so $\operatorname{dist}\left(T^{n} x T^{n} y\right) \leq B$. Hence

$$
\operatorname{diameter}\left(C_{j}^{n}\right)=\sup _{x, y \in C_{j}^{n}} \operatorname{dist}(x, y) \leq B(1 / \mu)^{n}
$$

Take $\lambda=\mu^{-1}$.

Because $T^{n}$ maps $C_{j}^{n}$ onto $X$, we have, for each $n$ and $j$,

$$
m\left(C_{j}^{n}\right) \cdot \sup _{C_{j}^{n}}\left|\operatorname{det} D T^{n}\right| \geq 1 \geq m\left(C_{j}^{n}\right) \inf _{C_{j}^{n}}\left|\operatorname{det} D T^{n}\right|,
$$

so

$$
\inf _{C_{j}^{n}} \frac{1}{\left|\operatorname{det} D T^{n}\right|} \leq m\left(C_{j}^{n}\right) \leq \sup _{C_{j}^{n}} \frac{1}{\left|\operatorname{det} D T^{n}\right|} .
$$

Since $R_{n}=\bigcup_{j=1}^{k^{n}} C_{j}^{n}$ we sum over $j$ and obtain

$$
\sum_{j} \inf _{C_{j}^{n}} \frac{1}{\left|\operatorname{det} D T^{n}\right|} \leq m\left(R_{n}\right) \leq \sum_{j} \sup _{C_{j}^{n}} \frac{1}{\left|\operatorname{det} D T^{n}\right|} .
$$

Since $1 /\left|\operatorname{det} D T^{n}(x)\right|=\exp \sum_{k=0}^{n-1}\left(T^{k} x\right)$, we can write $(1)$ as

$$
\sum_{j} \inf _{C_{j}^{n}} \exp \sum_{k=0}^{n-1} \phi\left(T^{k} x\right) \leq m\left(R_{n}\right) \leq \sum_{j} \sup _{C_{j}^{n}} \exp \sum_{k=0}^{n-1} \phi\left(T^{k} x\right) .
$$

We now show that the exponential growth rates of the quantities in (2) are the same. To do this, note that if $x, y$ are in the same set $C_{j}^{k}$, then $\operatorname{dist}(x, y) \leq B \lambda^{k}$ and hence there exists $K>0$ so that

$$
|\phi(x)-\phi(y)| \leq K \operatorname{dist}(x, y) \leq K B \lambda^{k}
$$

because $\phi$ is $C^{1}$ on each $C_{j}^{k}$. Consequently

$$
\begin{aligned}
\sum_{k=0}^{n-1} \phi\left(T^{k} x\right)-\sum_{k=0}^{n-1} \phi\left(T^{k} y\right) & \leq \sum_{k=0}^{n-1}\left|\phi\left(T^{k} x\right)-\phi\left(T^{k} y\right)\right| \leq \sum_{k=0}^{n-1} K B \lambda^{n-k} \\
& <K B \frac{1}{1-\lambda}=M
\end{aligned}
$$


independent of $n$. Then we have that

$$
\begin{aligned}
\lim _{n} \frac{1}{n} \log \frac{\sum_{j} \sup _{C_{j}^{n}} \exp \sum_{k=0}^{n-1} \phi\left(T^{k} x\right)}{\sum_{j} \inf _{C_{j}^{n}} \exp \sum_{k=0}^{n-1}\left(T^{k} x\right)} \\
\quad \leq \lim _{n} \frac{1}{n} \log \sum_{j}\left(\frac{\inf _{C_{j}^{n}} \exp \sum_{k=0}^{n-1} \phi\left(T^{k} x\right)}{\sum_{j} \inf _{C_{j}^{n}} \exp \sum_{k=0}^{n-1} \phi\left(T^{k} x\right)}\right) e^{M}=\lim _{n} \frac{M}{n}=0 .
\end{aligned}
$$

Since for any function $f$ we have that

$$
\sum_{j} \inf _{C_{j}^{n}} f \leq \sum_{j} \inf _{\Lambda_{j}^{n}} f \leq \sum_{j} \sup _{\Lambda_{j}^{n}} f \leq \sum_{j} \sup _{C_{j}^{n}} f
$$

we obtain from (2) that

$$
\log \alpha=\lim _{n} \frac{1}{n} \log m\left(R_{n}\right)=\lim _{n} \log \sum_{j} \sup _{\Lambda_{j}^{n}} \exp \sum_{k=0}^{n-1} \phi\left(T^{k} x\right) .
$$

To complete the proof it only remains to show that the right-hand side of $(3)$ is equal to $P(\phi)$. Note that it is equal to $P(\phi, \mathcal{U})$, where $\mathcal{U}=\left\{\Lambda \cap X_{1}, \ldots, \Lambda \cap X_{k}\right\}$. To find $P(\phi)=\lim _{\operatorname{diam}(\mathcal{U}) \rightarrow 0} P(\phi, \mathcal{U})$ we use the open covers $\mathcal{U}^{(n)}=\left\{\Lambda_{j}^{n}\right\}$ which have diameter $\left(\mathcal{U}^{(n)}\right) \leq B \lambda^{n}$. We show that $P\left(\phi, \mathcal{U}^{(n)}\right)=P(\phi, \mathcal{U})$. This is because, for fixed $m$,

$$
\begin{aligned}
\sum_{j}^{k^{n}} k^{m} \inf _{\Lambda_{j}^{n}} \exp \sum_{k=0}^{n-1} \phi\left(T^{k} x\right) & \leq \sum_{j}^{k^{m+n}} \sup _{\Lambda_{j}^{m+n}} \exp \sum_{k=0}^{n-1}\left(T^{k}\right) \\
& \leq \sum_{j=.}^{k^{n}} k^{m} \sup _{\Lambda_{j}^{n}} \exp \sum_{k=0}^{n-1} \phi\left(T^{k} x\right),
\end{aligned}
$$

which is obtained by replacing the sum over the $k^{m}$ sets $\Lambda_{j}^{m+n}$ which constitute a single $\Lambda_{j}^{n}$ by $k^{m}$ times the sup or inf over the $\Lambda_{j}^{n}$. But the right- and left-hand sides of (4) have the same exponential growth rate (in $n$ ) as the terms in (2).

The usefulness of Theorem 1 in estimating $\bar{\tau}$ comes from the variational inequality which says that for every $T$-invariant measure $\mu$ on $\Lambda$,

$$
h_{\mu}(T)+\int \phi d \mu \leq P(\phi)
$$

where $h_{\mu}(T)$ is the $\mu$-entropy of $T$ restricted to $\Lambda$. (See reference [1] for details.) Rather than enter into details we illustrate the theorem by estimating $\int \tau d \mu$ for a particular example.

Take $X=\{(x, y): 0 \leq x \leq 1,|y|<1\}$. Define $T: X \rightarrow \mathbf{R}^{2}$ by $T(x, y)=$ $(2 x(\bmod 1), f(x, y))$, where $f$ is a $C^{2}$ function on $X-\left\{(x, y): x=\frac{1}{2}\right\}$ with the properties that $f(x, 0)=0$ and $\partial f(x, y) / \partial y \geq \gamma>1$. Then $\Lambda=\{(x, y): y=0\}$ and $|\operatorname{det} D T(x, y)|=2|\partial f(x, y) / \partial y|$. In this setting, equality is attained in (5) for a unique measure $\mu^{*}$ on $\Lambda$ and $h_{\mu^{*}}(T)=\log 2$. Therefore

$$
\log \alpha=P(\phi)=h_{\mu^{*}}(T)-\int \log |\operatorname{det} D T| d \mu^{*} \geq \log 2-\sup _{\Lambda} \log \left[2 \frac{\partial f}{\partial y}(x, y)\right]
$$

so that $\alpha \geq \inf _{\Lambda}(\partial f / \partial y)^{-1}$. Denoting this inf by $s$, we obtain $\int \tau d \mu \geq s /(1-s)$. Similarly, $\int \tau d \mu \leq m /(1-m)$, where $m=\sup _{\Lambda}(\partial f / \partial y)^{-1}$. 
3. An example. In this section we consider one-parameter families $\left\{T_{\varepsilon}\right\}$ of transformations from $[0,1]$ to $\mathbf{R}$ and study the dependence of $\bar{\tau}$ on $\varepsilon$. In particular, we will describe a family $\left\{T_{\varepsilon}\right\}$ for which $\lim _{\varepsilon \rightarrow 0} \bar{\tau}_{\varepsilon} \varepsilon^{n}=+\infty$ for every $n \geq 0$. This says that the expected duration of the transients is large for $\varepsilon$ near 0 , and remains large for a substantial range of parameter values. This phenomenon has been observed numerically by Grebogi, et al. [2].

We begin by defining a function $T_{0}$ on $[0,1]$ which has very thin cusps at $x=\frac{1}{2}$ and $x=1$. To do this, select $\delta$ and $d_{1}>0$ and define

$$
T_{0}(x)= \begin{cases}1-\sqrt{-1 / \log \left(\frac{1}{2}-x\right)} & \text { for } \frac{1}{2}-d_{1}<x<\frac{1}{2} \\ (2-\delta) x & \text { for } 0 \leq x<\frac{1}{4}\end{cases}
$$

On $\left(\frac{1}{4}, \frac{1}{2}-d_{1}\right)$ define $T_{0}$ in such a way that $T_{0}$ is a $C^{2}$, concave up, monotone increasing function on $\left[0, \frac{1}{2}\right]$. In order to do this, $d_{1}$ must be quite small. For later convenience we record the fact that $d_{1}<\frac{1}{32}$. Define $T_{0}$ on $\left[\frac{1}{2}, 1\right]$ by translation: $T_{0}(x)=T_{0}\left(x-\frac{1}{2}\right)$. Note that the graph of $T_{0}(x)$ approaches the line $x=\frac{1}{2}$ in the same manner that $\exp \left(-1 / x^{2}\right)$ approaches the $x$-axis at $x=0$. Finally, define $T_{\varepsilon}(x)=(1+\varepsilon) T_{0}(x)$ for $\varepsilon \geq 0$.

Denoting $\left\{x: T_{\varepsilon}(x) \notin[0,1]\right\}=I \backslash T_{\varepsilon}^{-1}(I)$ by $A_{\varepsilon}$ we observe that there is an $\varepsilon_{0}>0$ such that $A_{\varepsilon} \subset\left[\frac{1}{2}-d_{1}, \frac{1}{2}\right] \cup\left[1-d_{1}, 1\right]$ for all $\varepsilon<\varepsilon_{0}$. Concerning $T_{\varepsilon}$ we show

Observation 1 . For each $\varepsilon>0, T_{\varepsilon}$ has an absolutely continuous conditionally invairant measure $\mu_{\varepsilon}$, the density of which, $d \mu_{\varepsilon} / d x=\rho_{\varepsilon}$, is a decreasing function of $x \in[0,1]$.

ProOf. The proof is a modification of that given in $[\mathbf{3}]$. Define an operator $Q_{\varepsilon}$ on $\mathcal{L}^{1}([0,1], m)$ by

$$
Q_{\varepsilon}(f)(x)=\frac{\sum_{T_{\varepsilon}(x)=x}\left(f(y) /\left|T_{\varepsilon}^{\prime}(y)\right|\right)}{\int_{0}^{1} \sum_{T_{\varepsilon}(y)=x}\left(f(y) /\left|T_{\varepsilon}^{\prime}(y)\right|\right) d x} .
$$

Nonnegative fixed points of $Q_{\varepsilon}$ are densities of absolutely continuous conditionally invariant measures. In $[\mathbf{3}]$ it is proved that there is a compact, convex set $C$ in $\mathcal{L}^{1}([0,1])$ which is mapped into itself by $Q_{\varepsilon}$. We observe that $Q_{\varepsilon}$ also preserves the set $D$ of decreasing functions on $[0,1]$, and so $Q_{\varepsilon}(C \cap D) \subset C \cap D$. Therefore $Q_{\varepsilon}$ has a fixed point in $C \cap D$. This fixed point is $\rho_{\varepsilon}$. The next two remarks provide bounds on $\rho_{\varepsilon}(x)$ which are independent of $\varepsilon$.

Observation 2. There is a number $b>1(2-\delta)$ such that for $\varepsilon<\varepsilon_{0}$,

$$
1 \geq \mu_{\varepsilon}\left(T_{\varepsilon}^{-1}(I)\right) \geq b .
$$

Proof. Denote $\mu_{\varepsilon}\left(T_{\varepsilon}^{-1}(I)\right)$ by $\alpha_{\varepsilon}$. This means $\alpha_{\varepsilon}=1-\int_{A_{\varepsilon}} \rho_{\varepsilon} d x$. Since $\rho_{\varepsilon}$ is decreasing and has integral $1,1 \geq \int_{0}^{1 / 4} \rho_{\varepsilon} d x \geq \rho_{\varepsilon}\left(\frac{1}{4}\right) \cdot \frac{1}{4}$, and so $\rho_{\varepsilon}\left(\frac{1}{4}\right) \leq 4$. Recall that $A_{\varepsilon} \subset\left[\frac{1}{2}-d_{1}, \frac{1}{2}\right] \cup\left[1-d_{1}, 1\right]$ when $\varepsilon>\varepsilon_{0}$ and that $d_{1}<\frac{1}{32}$. Then

$$
\int_{A_{\varepsilon}} \rho_{\varepsilon} d x \leq \rho_{\varepsilon}\left(\frac{1}{4}\right) \cdot m\left(A_{\varepsilon}\right) \leq 4 \cdot 2 d_{1} \leq \frac{1}{4}
$$

and so $\alpha_{\varepsilon} \geq 1-\frac{1}{4}$. For $b$ select any number satisfying $\frac{3}{4} \geq b>1 /(2-\delta)$.

Observation 3. For $\varepsilon<\varepsilon_{0}$, the functions $\rho_{\varepsilon}$ are uniformly bounded. 
PROOF. Since each $\rho_{\varepsilon}$ is a decreasing function of $x$, it is enough to find a bound on $\rho_{\varepsilon}(0)$ which is independent of $\varepsilon$. Because $\rho_{\varepsilon}$ is a fixed point of $Q$, we have that

$$
\rho_{\varepsilon}(0)=\left(\frac{\rho_{\varepsilon}(0)}{T_{\varepsilon}^{\prime}(0)}+\frac{\rho_{\varepsilon}(1 / 2)}{T_{\varepsilon}^{\prime}(1 / 2)}\right) \alpha_{\varepsilon}^{-1}=\left[\rho_{\varepsilon}(0)+\rho_{2}\left(\frac{1}{2}\right)\right] \frac{\alpha_{\varepsilon}^{-1}}{(2-\delta)} .
$$

Therefore, $b(2-\delta) \rho_{\varepsilon}(0) \leq \rho_{\varepsilon}(0)+\rho_{2}\left(\frac{1}{2}\right)$. As before, $\rho_{\varepsilon}\left(\frac{1}{2}\right) \leq 2$, and so $\rho_{\varepsilon}(0) \leq$ $2 /(b(2-\delta)-1)=\Delta$ for each $\varepsilon<\varepsilon_{0}$. We now have the estimate that $\int \tau_{\varepsilon} d \mu_{\varepsilon}=$ $\int \tau_{\varepsilon} \rho_{\varepsilon} d x \leq \Delta \int \tau_{\varepsilon} d x$, and therefore

$$
\bar{\tau}_{\varepsilon} \geq \frac{1}{\Delta} \int \tau_{\varepsilon} d \mu_{\tau}=\frac{1}{\Delta} \frac{\alpha_{\varepsilon}}{1-\alpha_{\varepsilon}} \geq \frac{1}{\Delta} \mu_{\varepsilon}\left(A_{\varepsilon}\right)^{-1} \geq \frac{1}{\Delta^{2}} m\left(A_{\varepsilon}\right)^{-1} .
$$

To complete the argument, note that $m\left(A_{\varepsilon}\right)=2 e^{-1 / \varepsilon^{2}} \leq 2 \varepsilon^{n}$ when $\varepsilon$ is small enough.

\section{REFERENCES}

1. R. Bowen Equilibrium states and the ergodic theory of Anosov diffeomorphisms, Lecture Notes in Math., vol. 470, Springer-Verlag, New York, 1973.

2. C. Grebogi, E. Ott and J. A. Yorke, Fractal basin boundaries, long lived chaotic transients and unstable-unstable pair bifurcation, Phys. Rev. Lett. 50 (1983), 935938.

3. G. Pianigiani and J. A. Yorke, Expanding maps on sets which are almost invariant: decay and chaos. Trans. Amer. Math. Soc. 252 (1979), 351366.

4. E. D. Yorke and J. A. Yorke, Metastable chaos: The transition to sustained chaotic behavior in the Lorenz model, J. Statist. Phys. 21 (1979), 263277.

Department of Mathematics, University of Maryland, College Park. MaryLAND 20742

Current address: Department of Mathematics, University of Cincinnati, Cincinnati. Ohio 45221 